\title{
Clinicopathological characteristics with EGFR, ALK, ROS1 genetic alternation and prognostic analysis of primary lymphoepithelioma- like carcinoma
}

\author{
Yinchen Shen, Fang Hu, Bo Zhang, Changhui Li, Xueyan Zhang, Baohui Han \\ Department of Pulmonary, Shanghai Chest Hospital, Shanghai Jiao Tong University, Shanghai 230032, China \\ Contributions: (I) Conception and design: B Han, Y Shen; (II) Administrative support: B Han, X Zhang; (III) Provision of study materials or patients: \\ Y Shen, B Zhang; (IV) Collection and assembly of data: Y Shen, F Hu, B Zhang; (V) Data analysis and interpretation: Y Shen, F Hu, C Li, B Han; (VI) \\ Manuscript writing: All authors; (VII) Final approval of manuscript: All authors. \\ Correspondence to: Baohui Han. Department of Pulmonary, Shanghai Chest Hospital, Shanghai Jiao Tong University, West Huaihai Road 241, Xuhui \\ District, Shanghai 230032, China. Email: 18930858216@163.com.
}

\begin{abstract}
Background: Primary lymphoepithelioma-like carcinoma (LELC) is a rare form of lung cancer, since genetic alternations participate in the carcinogenesis in lung cancer, whether critical driver genes such as ROS1 fusion, anaplastic lymphoma kinase (ALK) rearrangement and epidermal growth factor receptor $(E G F R)$ mutation play roles in LELC remains unclear.

Methods: We collected a total of 30 LELC samples for genetic and prognosis analysis retrospectively in Shanghai Chest Hospital, EGFR gene mutation, ALK rearrangement and ROS1 fusion status were extracted from digital database. Clinicopathological characteristics with genetic profiling and survival were analyzed.

Results: All samples appeared negative for genes (EGFR, ALK and ROS1) alternations detection. Female gender acted as an independently prognostic factor in poorer disease-free survival (DFS) $(\mathrm{P}=0.034)$, and tumors locate in the left lobe associate with worse DFS in univariate analysis (significant trend, $\mathrm{P}=0.051$ ), moreover, serum positive NSE level also indicate a shorter DFS after adjustment (significant trend, $\mathrm{P}=0.057$ ). Most of LELC are diagnosed at early stage, with $90.0 \%(27 / 30)$ patients obtained the opportunities for surgery.

Conclusions: Since no classical genetic alternations appeared in present study, more investigations of other genes distributions should be explored in the future for better understanding of this rare subtype of lung cancer. Serum positive neuron-specific enolase (NSE) level seemed to be a prognostic biomarker for DFS in LELC patients.
\end{abstract}

Keywords: Lymphoepithelioma-like carcinoma (LELC); genetic profiling; serum tumor biomarker; prognosis

Submitted Jun 10, 2019. Accepted for publication Sep 12, 2019.

doi: $10.21037 /$ tcr.2019.09.51

View this article at: http://dx.doi.org/10.21037/tcr.2019.09.51

\section{Introduction}

Lymphoepithelioma-like carcinoma (LELC) is a rare type of lung cancer, which was firstly reported in 1987, when Bégin et al. detected the Epstein-Barr virus (EBV) in a 40-yearold female, nonsmoker of South-East Asiatic origin (1). While LELC contains only $1.15 \%$ to $5.90 \%$ of lung cancers $(2,3)$, and less than 300 patients were reported in the past three years, details associated with this uncommon type require more description.

Primary pulmonary LELC was eliminated from the latest World Health Organization (WHO) lung cancer classification, and now is recognized as other and unclassified carcinomas (4). Published data indicated that LELC appeared more in young, non-smokers and no predilection with gender, nearly half patients were diagnosed at early stage (stage I and II), furthermore, majority of 
Table 1 Clinicopathological characteristics of 30 LELC patients LELC, lymphoepithelioma-like carcinoma

\begin{tabular}{|c|c|}
\hline Characteristics & Number (\%) \\
\hline \multicolumn{2}{|l|}{ Gender } \\
\hline Male & $16(53.3)$ \\
\hline Female & $14(46.7)$ \\
\hline \multicolumn{2}{|l|}{ Age } \\
\hline$>60$ & $14(46.7)$ \\
\hline$\leq 60$ & $16(53.3)$ \\
\hline \multicolumn{2}{|l|}{ Smoking history } \\
\hline Yes & $6(20.0)$ \\
\hline No & $23(76.7)$ \\
\hline Missing & $1(3.3)$ \\
\hline \multicolumn{2}{|c|}{ Primary tumor location } \\
\hline Left upper & $3(10.0)$ \\
\hline Left lower & $10(33.3)$ \\
\hline Right upper & $5(16.7)$ \\
\hline Right middle & $2(6.7)$ \\
\hline Right lower & $10(33.3)$ \\
\hline \multicolumn{2}{|l|}{ Tumor size $(\mathrm{cm})$} \\
\hline$>3$ & $13(43.3)$ \\
\hline$\leq 3$ & $16(53.3)$ \\
\hline Missing & $1(3.4)$ \\
\hline \multicolumn{2}{|l|}{ Tumor stage } \\
\hline 1 & $6(20.0)$ \\
\hline II & $8(26.7)$ \\
\hline III & $14(46.7)$ \\
\hline IV & $2(6.6)$ \\
\hline \multicolumn{2}{|c|}{ Progression at last follow-up } \\
\hline Yes & 7 (23.3) \\
\hline No & $21(70.0)$ \\
\hline Missing & $2(6.7)$ \\
\hline
\end{tabular}

this cancer type shared a longer survival compared with others in Asian populations (5-9), available treatments for LELC include complete resection, chemotherapy and radiotherapy if necessary (10). Meanwhile, baseline EBV DNA concentration, the serum albumin level, tumor stage and age of patients were all associated with prognosis in previous researches $(9,11,12)$, however, results from small sample size studies require further confirmations. Although genetic alternations were deeply investigated in lung cancer, driver oncogenes and related targeted drugs thrived in the past decades, few data were observed in this rare type, which appeals for more details, in order to understand this lung cancer subtype better.

Precision medicine spreads through the tumor diagnosis and treatment in recent years, for LELC, clinical features, pathological characteristics and gene mutations should be considered as a whole for a better vision. Herein, we collected 30 LELC patients for retrospective analysis in Southeaster China population, together with known drug-related gene targets such as epidermal growth factor receptor $(E G F R)$, anaplastic lymphoma kinase $(A L K)$ and ROS1 rearrangement, which may provide an appropriate insight in this rare type of lung cancer.

\section{Methods}

\section{Patients}

We totally recruited 30 LELC patients who were diagnosed and treated in Shanghai Chest Hospital between March 2011 and December 2017. Patients were excluded if metastasized from nasopharyngeal tumors. Detailed history data of these individuals were extracted from hospital digital database, such as patients' age, smoking status, tumor size, primary tumor location, staging information, peripheral blood tumor markers carcinoembryonic antigen (CEA), squamous cell carcinoma antigen (SCC), cytokeratin-19 fragment (CYFRA21-1), NSE, cancer antigen-125 (CA125), albumin, lactate dehydrogenase, immunohistochemistry (IHC) and gene detection results. EBV infection status was measured by in situ hybridization (ISH) for EBVencoded RNA (EBER) staining in tumor cells. EGFR gene mutation status was detected with amplification refractory mutation system (ARMS), ALK rearrangement was determined by IHC and ROS1 fusion status was detected with fluorescence in situ hybridization (FISH). Moreover, the tumor stage was performed according to the Eighth edition of American Joint Committee on Cancer (AJCC) staging system (13). Demographic and clinicopathological data of the study population were presented in Table 1. The study was approved by the Institutional Review Board (IRB) in Shanghai Chest Hospital, and informed consent was obtained from all patients before present study initiated. 


\section{End-points and follow-up}

All patients were recommended to receive sequential physical examination and image evaluation after initial treatments, for tumor recurrence monitoring. Disease-free survival (DFS) was defined as the interval time from surgical resection to the first confirmed relapse, or alive without recurrence at last contact, and time to death by any cause or last follow-up from operation was measured as overall survival (OS). Survival information was collected mainly by phone communication and outpatient visit. Clinical database was last updated in July 2018.

\section{Statistical analysis}

We utilized the Chi-square $\left(\chi^{2}\right)$ test or Fisher's exact test for clinicopathological characteristics comparison analysis, and Kaplan-Meier survival function with the method of log-rank test was performed in univariate relationships associated with DFS and OS. Furthermore, variants including age, gender, tumor location and size, tumor staging, chemotherapy and radiotherapy status were evaluated by fitting logistic regression in the survival analysis models, the hazard ratios were calculated with Cox proportional hazard regression models. All statistical analysis were carried out by the SPSS 17.0 statistical software (SPSS, Inc., Chicago, IL, USA) and GraphPad Prism 7, significance level was set at $\mathrm{P}<0.05$, as two-sides.

\section{Results}

\section{Clinical characteristics and treatment of LELC patients}

A total of 30 LELC patients were collected in the present study, of which $90.0 \%$ (27/30) underwent surgical resection in our center, 1 patient was diagnosed with fine needle aspiration and 2 patients were diagnosed in local hospitals. The mean age at diagnosis was 55.93 (range from 21 to 72), with relatively equivalent proportion between different genders (male $53.3 \%$ vs. female $46.7 \%$ ), most of patients were smoking-free population $(76.7 \%, 23 / 30)$, LELC appeared more frequently in the right lobe $(56.7 \%, 17 / 30)$ and only few patients existed metastasis at diagnosis $(6.7 \%$, $2 / 30$ ), which located in pleura and bones, respectively. Tumor stage balanced well between different genders ( $\mathrm{P}>0.05)$, for I-II stage in 14 patients ( 7 females and 7 males), and 16 patients were III-IV stage (7 females and 9 males). As for nodal status, 9 patients were N0 (4 females and 5 males), 21 patients with nodal metastasis (10 females and 11 males), and the $\mathrm{P}$ value $>0.05$. Twenty-five patients $(83.3 \%, 25 / 30)$ had received postoperative adjuvant chemotherapy and/or radiotherapy, of which pemetrexed $(31.6 \%, 6 / 19)$ or gemcitabine $(68.4 \%, 13 / 19)$ with cisplatin/ carboplatin contained $82.6 \%(19 / 23)$, while partial patients $(40.0 \%, 10 / 25)$ went through both treatment after surgery. During the follow-up, seven patients $(25.0 \%, 7 / 28)$ were identified as progression, which included five with lymph nodes, one with pleura, and one with bones metastasis.

\section{Serum tumor biomarkers, level of albumin and lactate dehydrogenase, EBV infection status, genetic alternations and IHC results profiles}

We selected five serum tumor biomarkers in present study, which included CEA, SCC, CYFRA21-1, NES and CA125. All serum data were collected before surgery or the biopsy. The positive rate of these biomarkers was 3.6\% (1/28) for CEA, $10.7 \%$ (3/28) for SCC, $25.0 \%$ (7/28) for CYFRA21-1, 10.7\% (3/28) for NSE and $7.1 \%(2 / 28)$ in CA125. The albumin and lactate dehydrogenase level were detected in total 28 patients, and all albumin level were negative, while positive rate for lactate dehydrogenase was $10.7 \%(3 / 28)$. Besides, EB virus infection status was obtained in $33.3 \%(10 / 30)$ patients and $90.0 \%(9 / 10)$ were positive in the set. As for genetic mutations detection in this study, EGFR, ALK and ROS1 status were available in $63.3 \%$ (19/30), $53.3 \%(16 / 30)$ and 40\% (12/30), respectively. All the gene alternations were negative in this study. The status of thyroid transcription factor-1 (TTF-1) was detected in $93.3 \%(28 / 30)$ samples, only one $(3.6 \%, 1 / 28)$ appeared positive, and cytokeratin 7 (CK7) was detected in $60.0 \%$ $(18 / 30)$ samples, all of them showed negative in present study, while cytokeratin 5/6 (CK5/6) was available in $80.0 \%$ (24/30) specimens, and $95.8 \%(23 / 24)$ harbored positive results. $\mathrm{P} 40$ was also investigated and all the detected samples $(76.7 \%, 23 / 30)$ presented positive $(100 \%, 23 / 23)$.

\section{Univariate and multivariate analysis of outcomes predictors in primary LELC}

The median follow-up for outcomes analysis in this cohort was 27 months, 3 patients lost the follow-up, and $90 \%(27 / 30)$ population fulfilled the survival information collection. Patients' clinicopathological characteristics and blood tumor markers (CEA, SCC, CYFRA21-1, NES, CA125), level of albumin and lactate dehydrogenase were included in analysis with DFS and OS, we found female 


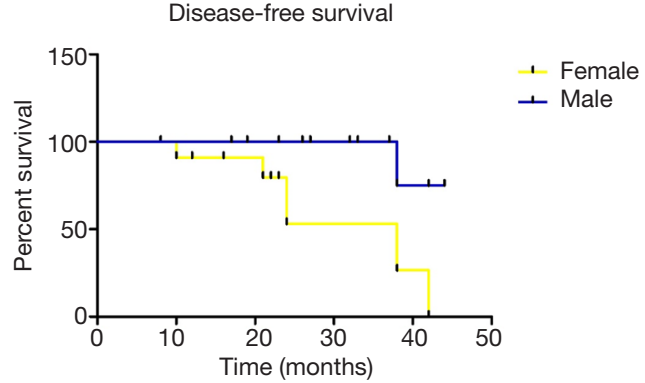

Figure 1 Disease-free survival in different genders of LELC. LELC, lymphoepithelioma-like carcinoma.

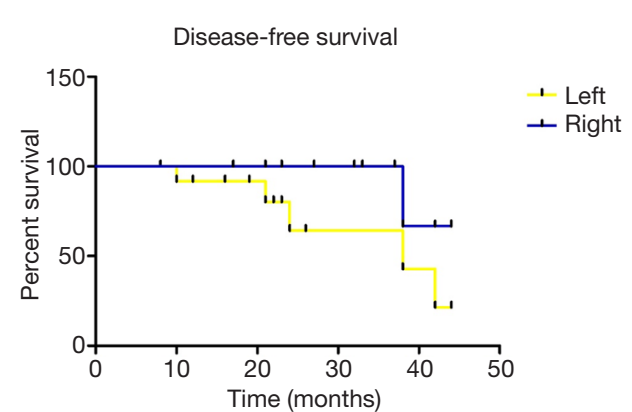

Figure 2 Disease-free survival in different tumor locations of LELC. LELC, lymphoepithelioma-like carcinoma.

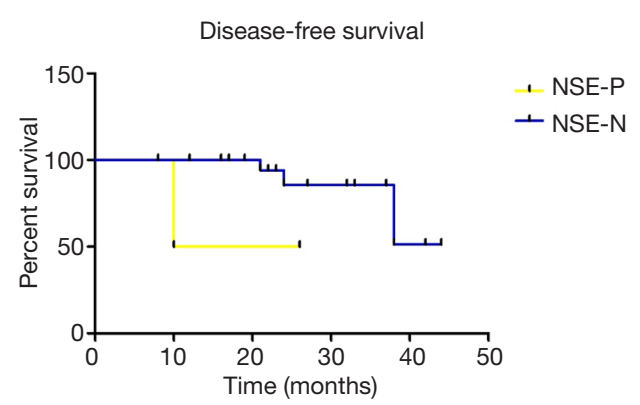

Figure 3 Disease-free survival in different NSE levels of LELC. NSE, neuron-specific enolase; LELC, lymphoepithelioma-like carcinoma.

was more likely to obtained a shorter DFS (38 months vs. not reached, $\mathrm{P}=0.008$ ) (Figure 1), and tumor located in left lobe or positive NSE level seemed to indicate a poorer DFS (Figures 2 and 3), although only appeared a significant trend ( $\mathrm{P}=0.051$ for tumor location, $\mathrm{P}=0.086$ for NSE level). Besides, no other characteristics or blood biomarkers acted as prognostic in LELC patients, both for DFS and OS analysis. And chemotherapy regimens did not appear significantly different between patients in survival outcomes ( $\mathrm{P}=0.974$ for DFS, $\mathrm{P}=0.724$ for OS). In the multivariate analysis, we selected gender, tumor location, peripheral NSE level, nodal status and tumor staging in relation to patients' DFS and OS. The female gender also achieved significance after adjustment, and acted independently prognostic for shorter DFS ( $\mathrm{P}=0.034)$, furthermore, NSE level appeared a significant trend in DFS, with NSE positive indicating a shorter DFS $(\mathrm{P}=0.057)$ (Table 2), no other associations were discovered in the analysis, while for OS evaluation, no significant differences were discovered.

\section{Discussion}

In this retrospective study, we conducted a comprehensive study for a rare type of lung cancer, which in order to overview the clinical characteristics, peripheral blood tumor biomarkers, genetic alternations and IHC results profile, and potential prognostic variants for LELC. Since published data was rare in this cancer subtype, details of LELC remains controversial in some respects. As precision medicine sparkled thriving researches in tumor diagnosis and treatment, we also required more data in this uncommon cancer type.

LELC is a unique histological type of lung cancer, and appears specific clinicopathological characteristics from other lung malignancies. Reported researches indicated that LELC occurred more in young patients (14), the patients in our cohort was diagnosed with a mean age at 55.93 (range from 21 to 72), which was among the average reported results $(11,14)$, besides, non-smokers were more common in this population, Wang et al. recruited 42 LELC patients, and $69.0 \%$ were smoking-free population (15), we achieved a $76.7 \%$ non-smokers here, which was consistent with previous studies $(11,14,16,17)$. Gender difference was generally not discovered in LELC (18), and our data also found that the incidence was similar between males and females (53.3\% vs. 46.7\%). LELC seemed more frequently in the right lobe, our findings reported $56.7 \%(17 / 30)$ located in the right, similar to the results presented by Yu et al. $(52.9 \%, 46 / 87)$ (19). Only two patients $(6.7 \%)$ existed distant metastasis when diagnosed, which was obviously lower than other subtypes of lung cancer, Liang et al. also reported 5.8\% (3/52) patients with metastasis (11), which was similar in different studies (17). Moreover, some researches showed nearly no metastasis in LELC $(2,19,20)$ at diagnosis, which may explain partially the better prognosis in pulmonary LELC than other cancer 
Table 2 Multivariate analysis of outcomes predictors in primary LELC

\begin{tabular}{lccc}
\hline Prognostic characteristics & P value & Hazard ratio & $95 \% \mathrm{Cl}$ \\
\hline Gender & 0.028 & 0.062 & $0.005-0.720$ \\
Tumor location & 0.709 & 0.478 & $0.010-23.020$ \\
NSE status & 0.057 & 17.705 & $0.915-342.716$ \\
Nodal stage & 0.732 & 0.48 & $0.007-32.419$ \\
Tumor stage & 0.748 & 0.479 & $0.005-42.524$ \\
\hline
\end{tabular}

LELC, lymphoepithelioma-like carcinoma; NSE, neuron-specific enolase.

subtypes suffered in lung.

As for the serum biomarkers, CYFRA21-1 (25.0\%, $7 / 28$ ) seemed the most common positive tumor marker for LELC in present study, followed by SCC, NSE (both were $10.7 \%, 3 / 28)$ and $\operatorname{CA} 125$ (7.1\%, 2/28), which was consistent with previous study $(16,17)$, furthermore, NSE appeared a significantly statistical trend for DFS in multivariate analysis, while others did not share this in survival evaluation, since the sample size was relatively small, magnitude of this discovery remained more data to achieve a robust conclusion, we noticed only 3 patients owned abnormal NSE level at present study, and 1 patient with stage II and 2 patients with stage III, all three patients with nodal metastasis, the bias may influence the result to some extent, considering NSE was a widely used tumor biomarker for diagnosis and treatment in lung cancer, further studies might draw more attention at this. We also collected the IHC results of LELC, of which TTF-1 expression was nearly negative $(3.6 \%, 1 / 28)$, and CK7 showed no positive, while most samples harbored positive CK5/6 and P40 expression, of which were 95.8\% and $100 \%$, respectively. Reported data presented similar results, with a $100 \%$ positive for CK5/6 and $97.1 \%$ negative for CK $7,100 \%$ negative for TTF-1 (15). Since TTF-1 and CK5/6 were recommended in IHC marker panel for differentiation between adenocarcinoma (AC) and SCC, the predilection of LELC IHC results was more likely to imitate SCCs, which indicated whether more biological behavior were shared between these two subtypes of lung cancer, few data referred to this appealed for future investigation.

Driver genes associated lung cancer always required specific targeted therapy, such as EGFR-tyrosine kinase inhibitor (TKI) or ALK/ROS1 kinases inhibitors (21-23), driver genes detection before treatments were strongly recommended in different guidelines in order to obtain the greatest benefit for patients, however, the gene alternations status for LELC were still in veil to some extent, reported data was rare at present. Herein, we retrospectively evaluate the driver genes outline for LELC, interestingly, all samples showed negative results for all three genes (EGFR, ALK and ROS1). Wang et al. also recruited a 42 patients cohort for genes detection ( $E G F R$ and $A L K$ ), the results were consistent with ours, only 1 patient $(2.4 \%, 1 / 42)$ harbored EGFR L858R mutation and none of the 42 patients harbored ALK rearrangement (15), while other studies also presented different $E G F R$ gene mutation frequency, from $9.1 \%(\mathrm{n}=11)$ to $17.4 \%(\mathrm{n}=46)(5,24)$, since the $E G F R$ mutation status was evaluated by ARMS in our center, only common mutation types (such as L858R, 19 del etc.) were detected, then uncommon subtypes of alternations located in other exons were unclear with ARMS, which led to a lower mutation frequency compared to previous studies evaluated by sanger sequencing, majority mutations of EGFR were in exon 21 but without L858R in Chang et al. report (5), for instance. Different studies also showed ALK rearrangement was rare in LELC, nearly no ALK alternations in this subtype $(15,25)$, which suggested ALK might not be a driver gene in LELC tumorgenesis. However, all the results derived from small cohort, we require more data in the future to confirm these gene alternations in LELC and draw more precise conclusions.

Generally, LELC owned a better survival than other types of lung cancer, with most patients distributed in early stages and available for surgery (9), here $90.0 \%(27 / 30)$ patients underwent surgery in our study, and only 2 patients (6.7\%) suffered distant metastasis at diagnosis. Female gender appeared a worse DFS in both univariate and multivariate analysis of outcomes, while similar results were not mentioned in previous researches, considering the small sample size, the result should be confirmed further $(9,17)$, whether gender acted as a pivotal role in DFS was still controversial, and most attributed to the data deficiency. Besides, there existed several limitations in present study 
such as relatively small sample size and no abundant genetic or immune biomarkers, the scarce data may not reflect comprehensive gene alternations in LELC, besides, immunotherapy has been implemented in lung cancer $(26,27)$, and PD-L1 may act as a potential biomarker for LELC in prognosis prediction (21), then larger sample size and intensive analysis of genetic or protein level variations should be investigated in the future. Since next generation sequencing (NGS) was widely used in cancer research, a deeper and precise insight may rely on this, to bring us a step closer for this rare subtype of lung cancer.

\section{Conclusions}

In conclusion, LELC was a rare while with an ideal prognosis subtype in lung cancer, characteristics of this population was inclined to younger and non-smokers, female gender acted as an independent prognostic factor in worse DFS, and serum NSE level might be a potential tumor marker for survival prediction. Common driver genes of lung AC seemed uncommon in this rare subtype, and more investigations could provide a better understanding of LELC in the future.

\section{Acknowledgments}

The authors would like to acknowledge the support from the Department of Pathology of Shanghai Chest Hospital. We also thank the patients and their families for their contributions to this study.

Funding: None.

\section{Footnote}

Conflicts of Interest: The authors have completed the ICMJE uniform disclosure form (available at http://dx.doi. org/10.21037/tcr.2019.09.51). The authors have no conflicts of interest to declare.

Ethical Statement: The authors are accountable for all aspects of the work in ensuring that questions related to the accuracy or integrity of any part of the work are appropriately investigated and resolved. The study was conducted in accordance with the Declaration of Helsinki (as revised in 2013). The study was approved by the Institutional Review Board (IRB) in Shanghai Chest Hospital [No. KS(Y)1982], and informed consent was obtained from all patients before present study initiated.
Open Access Statement: This is an Open Access article distributed in accordance with the Creative Commons Attribution-NonCommercial-NoDerivs 4.0 International License (CC BY-NC-ND 4.0), which permits the noncommercial replication and distribution of the article with the strict proviso that no changes or edits are made and the original work is properly cited (including links to both the formal publication through the relevant DOI and the license). See: https://creativecommons.org/licenses/by-nc-nd/4.0/.

\section{References}

1. Bégin LR, Eskandari J, Joncas J, et al. Epstein-Barr virus related lymphoepithelioma-like carcinoma of lung. J Surg Oncol 1987;36:280-3.

2. Liang R, Chen TX, Wang ZQ, et al. A retrospective analysis of the clinicopathological characteristics of large cell carcinoma of the lung. Exp Ther Med 2015;9:197-202.

3. Pardo J, Martinez-Peñuela AM, Sola JJ, et al. Large cell carcinoma of the lung: an endangered species? Appl Immunohistochem Mol Morphol 2009;17:383-92.

4. Travis WD, Brambilla E, Nicholson AG, et al. The 2015 World Health Organization Classification of Lung Tumors: Impact of Genetic, Clinical and Radiologic Advances Since the 2004 Classification. J Thorac Oncol 2015;10:1243-60.

5. Chang YL, Wu CT, Shih JY, et al. Unique p53 and epidermal growth factor receptor gene mutation status in 46 pulmonary lymphoepithelioma-like carcinomas. Cancer Sci 2011;102:282-7.

6. Han AJ, Xiong M, Gu YY, et al. Lymphoepitheliomalike carcinoma of the lung with a better prognosis. A clinicopathologic study of 32 cases. Am J Clin Pathol 2001;115:841-50.

7. Ho JC, Wong MP, Lam WK. Lymphoepithelioma-like carcinoma of the lung. Respirology 2006;11:539-45.

8. Ngan RK, Yip TT, Cheng WW, et al. Clinical role of circulating Epstein-Barr virus DNA as a tumor marker in lymphoepithelioma-like carcinoma of the lung. Ann N Y Acad Sci 2004;1022:263-70.

9. He J, Shen J, Pan H, et al. Pulmonary lymphoepitheliomalike carcinoma: a Surveillance, Epidemiology, and End Results database analysis. J Thorac Dis 2015;7:2330-8.

10. Wong JF, Teo MC. Case report: lymphoepithelial-like carcinoma of the lung--a chronic disease? World J Surg Oncol 2012;10:91.

11. Liang Y, Wang L, Zhu Y, et al. Primary pulmonary lymphoepithelioma-like carcinoma: fifty-two patients with 
long-term follow-up. Cancer 2012;118:4748-58.

12. Xie M, Wu X, Wang F, et al. Clinical Significance of Plasma Epstein-Barr Virus DNA in Pulmonary Lymphoepithelioma-like Carcinoma (LELC) Patients. J Thorac Oncol 2018;13:218-27.

13. Detterbeck FC, Boffa DJ, Kim AW, et al. The Eighth Edition Lung Cancer Stage Classification. Chest 2017;151:193-203.

14. Sun YH, Lin SW, Hsieh C, et al. Treatment outcomes of patients with different subtypes of large cell carcinoma of the lung. Ann Thorac Surg 2014;98:1013-9.

15. Wang L, Lin Y, Cai Q, et al. Detection of rearrangement of anaplastic lymphoma kinase (ALK) and mutation of epidermal growth factor receptor (EGFR) in primary pulmonary lymphoepithelioma-like carcinoma. J Thorac Dis 2015;7:1556-62.

16. Lin L, Lin T, Zeng B. Primary lymphoepithelioma-like carcinoma of the lung: An unusual cancer and clinical outcomes of 14 patients. Oncol Lett 2017;14:3110-6.

17. Jiang WY, Wang R, Pan XF, et al. Clinicopathological features and prognosis of primary pulmonary lymphoepithelioma-like carcinoma. J Thorac Dis 2016;8:2610-6.

18. Mo Y, Shen J, Zhang Y, et al. Primary lymphoepitheliomalike carcinoma of the lung: distinct computed tomography features and associated clinical outcomes. J Thorac Imaging 2014;29:246-51.

19. Yu X, Wen Y, Qin R, et al. Prognosis and distribution of lymph nodes metastases in resectable primary pulmonary lymphoepithelioma-like carcinoma: A large cohort from a single center. Thorac Cancer 2018;9:360-7.

20. Yu XY, Zhang XW, Wang F, et al. Correlation and

Cite this article as: Shen Y, Hu F, Zhang B, Li C, Zhang $\mathrm{X}$, Han B. Clinicopathological characteristics with EGFR, $A L K$, ROS1 genetic alternation and prognostic analysis of primary lymphoepithelioma-like carcinoma. Transl Cancer Res 2019;8(6):2350-2356. doi: 10.21037/tcr.2019.09.51 prognostic significance of PD-L1 and P53 expression in resected primary pulmonary lymphoepithelioma-like carcinoma. J Thorac Dis 2018;10:1891-902.

21. Soria JC, Ohe Y, Vansteenkiste J, et al. Osimertinib in Untreated EGFR-Mutated Advanced Non-Small-Cell Lung Cancer. N Engl J Med 2018;378:113-25.

22. $\mathrm{Wu} \mathrm{YL,} \mathrm{Zhou} \mathrm{C}, \mathrm{Hu} \mathrm{CP}$, et al. Afatinib versus cisplatin plus gemcitabine for first-line treatment of Asian patients with advanced non-small-cell lung cancer harbouring EGFR mutations (LUX-Lung 6): an open-label, randomised phase 3 trial. Lancet Oncol 2014;15:213-22.

23. Hida T, Nokihara H, Kondo M, et al. Alectinib versus crizotinib in patients with ALK-positive non-small-cell lung cancer (J-ALEX): an open-label, randomised phase 3 trial. Lancet 2017;390:29-39.

24. Tam IY, Chung LP, Suen WS, et al. Distinct epidermal growth factor receptor and KRAS mutation patterns in non-small cell lung cancer patients with different tobacco exposure and clinicopathologic features. Clin Cancer Res 2006;12:1647-53.

25. Wong DW, Leung EL, So KK, et al. The EML4-ALK fusion gene is involved in various histologic types of lung cancers from nonsmokers with wild-type EGFR and KRAS. Cancer 2009;115:1723-33.

26. Reck M, Rodríguez-Abreu D, Robinson AG, et al. Pembrolizumab versus Chemotherapy for PD-L1Positive Non-Small-Cell Lung Cancer. N Engl J Med 2016;375:1823-33.

27. Borghaei H, Paz-Ares L, Horn L, et al. Nivolumab versus Docetaxel in Advanced Nonsquamous Non-Small-Cell Lung Cancer. N Engl J Med 2015;373:1627-39. 